\begin{tabular}{|c|c|}
\hline & $\begin{array}{l}\text { International Journal of Trend in Scientific } \\
\text { Research and Development (IJTSRD) }\end{array}$ \\
\hline 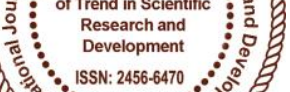 & International Open Access Journal \\
\hline 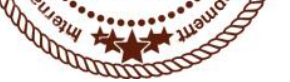 & ISSN No: 2456 - 6470 | www.jitsrd.com | Volume - 2 | Issue -3 \\
\hline
\end{tabular}

\title{
Approaches of Pattern Recognition in Analysing the Neurological Disorders: A Review
}

\author{
Mrs. Rashmi Somshekhar \\ Research Scholar, Department of Computer Science, AWUV, Vijayapura, Karnataka, India
}

\begin{abstract}
Neurological disorder is mainly concerned with the body nervous system. For most of the neurological disorders diagnostic imaging generates a particular pattern for identifying the different anomalies. Pattern Recognition which is used as tool for extracting the features from a lot of noisy data by which the different patterns are recognized by using different approaches like Template matching, Statistical, Syntactic or Structural and neural network based techniques. This paper Reviews the different approaches of Pattern Recognition that are likely suitable for recognizing the particular pattern of the neurological disorders . All these techniques help in decision support to diagnose the treatment of illnesses.
\end{abstract}

Keywords: Pattern Recognition, Template matching, Statistical, Syntactic or Structural, neural network and Neurological disorders

\section{INTRODUCTION}

The mechanism of recognizing and then sorting different pieces of information and making decisions or predictions based on the collected information will be very interesting both in humans and in electronics. Pattern Recognition is one of the tools in Image Processing that helps in making important decisions. It is an automated analysis of collected attributes of objects, events, etc, to classify them into categories [1]. Pattern Recognitions provides solution to various problems in real life applications like bioinformatics, document classification, image analysis, data mining, industrial automation, biometric recognition, remote sensing, handwritten text analysis, medical diagnosis, speech recognition, GIS and many more[2].The more accurately you recognize the pattern the more accurate will be your decisions. To recognize the patterns we have different approaches or models like Statistical Techniques, Structural Techniques, Template Matching and Neural Network based techniques [3].The Pattern recognition plays a critical role when applied to medical imaging by fully automating process of abnormality detection and thus supporting the development of computer aided diagnosing system (CAD)[3].Using these approaches in Neurological disorders which will helps the physician to rule out or confirm the diseases.

There are different imaging tools, including imaging technologies to image the nervous system. Many Researchers and physicians use a variety of diagnostic imaging techniques to analyses, detect, manage, and treat neurological disease [4]. In this paper we are trying to analysis such an approach for diagnosing neurological disorders which acts as a decision supporting tool that will help in identifying the anomalies with more accuracy.

\section{PATTERN RECOGNITION:}

Pattern is defined as description of an object. The object might be anything; you are trying to search a pattern in that object. For example, a pattern could be a fingerprint image, a handwritten cursive word, a human face, or a speech signal. Pattern recognition as a classification of input data via extraction important features from a lot of noisy data [5].The basic functions of a pattern recognition system are to detect and extract common features from the patterns describing the objects that belong to the same pattern class, and to recognize this pattern in any new 
environment and classify it as a member of one of the pattern classes under consideration. There are two methods of recognizing/ classifying the patterns based on the required specifications. Supervised learning/Classification: It is an automatic system that confirms the patterns described by the users on a representation defined by $\mathrm{him} / \mathrm{her}$ and it is done by automatic classification followed by an evaluation. Unsupervised learning/clustering: It automatically suggests the presence of unspecified patterns (regularities) in the observations [3].

\section{A. Pattern Recognition Design:}

System designing are often very complicate due to the presence of many unknown complexities. However, to understand the design of the system, we need to first understand the different components [6].First step, Sensing: Sensors in a system is what receives the input data; it may vary system to system depending upon the purpose. Examples are transducers such as camera or a microphone [7] Second step, Segmentation: Once we receive the input data we need to separate it . The toughest problem of segmentation in pattern recognition is because a lot of the patterns tends to overlap and intermingle. In medical image analysis image segmentation helps in identifying the boundaries of objects such as organs or abnormal regions (e.g. tumors) in images [20].The segmentation result makes it possible for shape analysis, detecting volume change, and making precise treatment plans [10]. Third step, Feature Extraction and Classification: Finding distinguishing features those are invariant to any transformations of the data and using features and learned models to assign a pattern to a category.

Fourth step, Post Processing: The post-processor uses the output of the classifier to decide on the recommended action on the data.

The image shows the various components of a Patten recognition system.

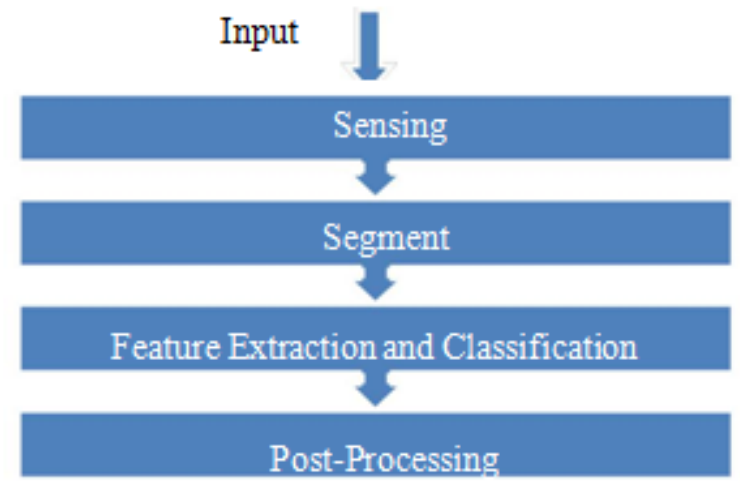

Decision

Fig1: Process of pattern Recognition System

The design of pattern recognition also involves the repetition of the design cycle which contains different activities. The different cycles involved are; Data Collection: Collecting training and testing data.

Feature and Model Choices: Making choice of distinguishable features is a very critical step. The Prior knowledge data will help in selecting the right features. Training. This phase helps to determine how we can learn the rule from data. Supervised learning: a teacher provides a category label or cost for each pattern in the training set. Unsupervised learning: the system forms clusters or natural groupings of the input patterns. Reinforcement learning: no desired category is given but the teacher provides feedback to the system such as the decision is right or wrong [8]. The process of using the data to determine the classifier is known as training the classifier.

Evaluation: To measure the performance of the system evaluation is important and it also indicates if there is any room for improvement [7].

The image shows an example of the design cycle for a pattern recognition system. 


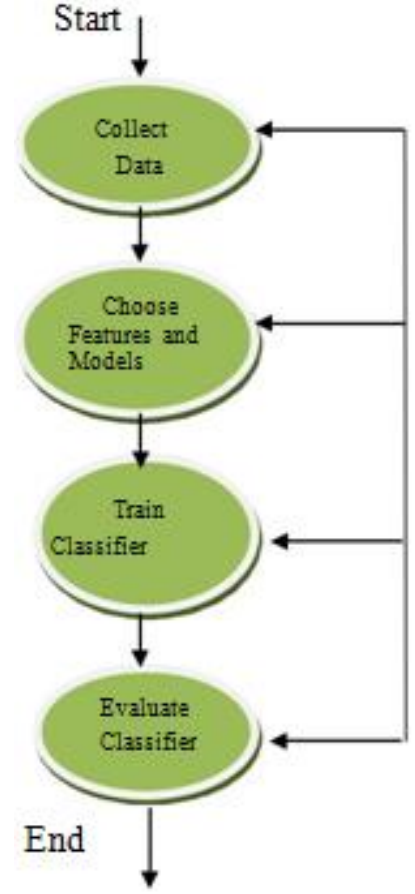

Fig2: Design Cycle

\section{APPROACHES/MODELS}

\section{RECOGNITION SYSTEM:}

The following are some approaches that help in designing the pattern recognition system.

- Template Matching.

- Statistical methods.

- Syntactic methods.

- Neural networks.

Template Matching: It is mainly the prototype of the pattern. It is the most efficient and simple technique which gives very good results and response. Whatever pattern you are recognizing should match to the template, it means that the prototype .It is used to detect simultaneously any two objects or entities; it may be curves, points or shapes etc [10].Example for neurological disorder: The processing of paediatric neuro imaging data is a challenge. For that, the approaches construct reference data (templates) by averaging brain images from a control group of subjects, or by creating custom templates from the group under study [18].

Statistical Pattern Recognition: In the statistical approach, the patterns are described as random variables, from which class densities can be inferred. Classification is done based on the statistical modeling of data. It is described in terms of feature sets. The effectiveness of the feature set is determined by how well patterns from different classes can be separated i.e., there is a proper interclass distance
[11].However, the Statistical pattern recognition deals only with the features without considering the relations between features. [12].one of the example of statistical methods was used to classify the clinical cases of cognitive impairment based on electroencephalograms (EEG). A classifier was created for each possible pair of groups using statistical pattern recognition (SPR) [21].

Syntactic model: Syntactic pattern recognition uses the structural information for classification and description. Grammars can be used to create a definition of the structure of each pattern class. They are also called as structural models and are based on the relation between the features. It is often difficult to classify patterns that contain a large number of features [13].For Example patients with probable Alzheimer's disease (AD) was evaluated with the ability to use syntactic cues significantly more intact . These findings are consistent with a modular theory of grammar and of mental functions more generally [19].

Neural Network: Neural computing is based on the way by which biological neural system store and manipulates information. Many algorithms have been designed to work with neural network. Many types of neural networks like RBF (Radical Basis Function), Feed Forward Network, PCA (Principle Component Analysis), SVM (Support Vector Machine).

\section{Table1: Model/Approaches in Pattern} Recognition.

\begin{tabular}{|l|l|l|l|}
\hline Model or & \multicolumn{2}{|l|}{$\begin{array}{l}\text { Recognition } \\
\text { Representation }\end{array}$} & Criterion \\
\hline Approach & & Cunction & \\
\hline $\begin{array}{l}\text { Template } \\
\text { matching }\end{array}$ & $\begin{array}{l}\text { Correlation, } \\
\text { Distance }\end{array}$ & $\begin{array}{l}\text { Samples, } \\
\text { Pixels, }\end{array}$ & $\begin{array}{l}\text { Classification } \\
\text { error }\end{array}$ \\
\hline & Measure & Curves & \\
\hline $\begin{array}{l}\text { Statistical } \\
\text { approach }\end{array}$ & $\begin{array}{l}\text { Discriminat } \\
\text { e Function }\end{array}$ & Features & $\begin{array}{l}\text { Classification } \\
\text { error }\end{array}$ \\
\hline $\begin{array}{l}\text { Structural } \\
\text { approach }\end{array}$ & $\begin{array}{l}\text { Rules/ } \\
\text { Grammar }\end{array}$ & Primitive & $\begin{array}{l}\text { Acceptance } \\
\text { error }\end{array}$ \\
\hline $\begin{array}{l}\text { Neural } \\
\text { Network }\end{array}$ & $\begin{array}{l}\text { Network } \\
\text { Function }\end{array}$ & $\begin{array}{l}\text { Samples, } \\
\text { Pixels, }\end{array}$ & $\begin{array}{l}\text { Mean square } \\
\text { error }\end{array}$ \\
\hline approach & & Curves & \\
\hline
\end{tabular}

A. Pattern Recognition Approaches used in Clinical Medicine for analysis neurological disorders:

[1] Neural Networks

[2] Bayesian Belief Networks 
[3] Support Vector Machines(SVM)

[4] Tree Classifiers

a. Multiclassifiers: A combination of above Artificial Neural Networks: A simulation of model in solving problems in pattern recognition, optimization etc.

\section{Neurological disorders:}

The Neurological disorders are the diseases of the brain, spine and the nerves that connect them. They are the disorders concerned with the body nervous system. We find more than 600 diseases of the nervous system. The Diagnostic tests and procedures are vital tools that help physicians confirm or rule out the presence of a neurological disorder or other medical condition [13]. Imaging is performed in various modalities, such as MRI, CT, ultrasound, positron emission tomography (PET), etc. The Researchers and physicians use a variety of diagnostic imaging techniques to analyses to detect, manage, and treat the neurological disease. [6],[16].

\section{Pattern Recognition as Decision support in Neurological disorders:}

The increasing interest in the development of pattern recognition based medical imaging that has helped to solve the problems by early diagnosis. We make systems that we call 'decision support' that only gives opinion to physician. Making decision will face lot of uncertainty and dealing with uncertainties is a common problem in pattern recognition [7].In medical imaging the process of abnormality detection and thus supported by the development of computer aided diagnosing system (CAD) [17]. There are some examples of Pattern Recognition in Medical Decision Support. Interpreting 1D data such as in ECG, EEG. Interpreting 2D data: detecting cells, tumors or any other abnormalities in any $\mathrm{x}$-ray, MR, tomography etc .Sequence processing in genetic data. Processing of any collected numerical data such as blood test results. Processing any collected non-numeric data such as patient history, doctor interpretations and reports. Using more than one of these together to use in decisions and treatment of an illness [1],[14]. These are some pattern recognition approaches that have shown their effectiveness in medical diagnosis. Using the decision supporting tool called as pattern recognition approaches it will help the physician to confirm the anomalies in neurological disorders.

\section{CONCLUSIONS:}

Analyzing and understanding the disease process at its early stages with more accurately needs medical imaging process, which plays a vital role in early diagnosis. By applying approaches of pattern recognition diagnosis methods in medical imaging can help solve the problems. In this paper, we are trying to propose decision support tools of image processing for recognizing the particular pattern for the disorders. By this it will help to train the system to identify the disorders with more accuracy and today, Pattern Recognition methods are accepted as useful tools in the service of M.D.'s as consultants in clinical decision making.

\section{REFERENCES}

1. Neşe Yalabık : Medical Applications of Pattern Recognition. HIBIT'10,Antalya, April 2010.

2. Seema Asht and Rajeshwar Dass: Pattern Recognition Techniques: A Review. International Journal of Computer Science and Telecommunications, Volume 3, Issue 8, August 2012.

3. Anil K. Jain: Statistical Pattern Recognition: A Review IEEE transactions on Pattern Analysis and Machine Intelligence, vol. 22, no. 1, january 2000.

4. http://www.ninds.nih.gov : Neurological Diagnostic Tests and Procedures. NINDS. March 1, 2005, NIH Publication No. 05-5380.

5. Gonzalez, R.C.Thomas and M.G: Syntactic Pattern Recognition: an Introduction. Addison Wesley, Reading, MA, 1978.

6. https://www.projectrhea.org : Introduction To Pattern Recognition and Classification .Rhea projectrhea.org.

7. Shahin Ara Begum and O. Mema Devi: Fuzzy Algorithms for Pattern Recognition in Medical Diagnosis. Assam University Journal of Science \& Technology: ISSN 0975-2773, Physical Sciences and Technology, Vol. 7 Number II,1-12, 2011.

8. S. P. Shinde and V.P.Deshmukh: Implementation of Pattern Recognition Techniques and Overview of its Applications in Various areas of Artificial Intelligence. International Journal of Advances in Engineering \& Technology, Sept 2011. 
9. Xiaolei Huang and Gavriil Tsechpenakis: Medical Image Segmentation. Computer Science and Engineering Department, Lehigh University and Center for Computational Sciences, University of Miami.

10. Rajleen Kaur: An Overview of Pattern Recognition Applications and Its Approaches: A Review. International Journal of Science and Research (IJSR), ISSN (Online): 2319-7064, Impact Factor (2012): 3.358.

11. Priyanka Sharma and Manavjeet Kaur:Classification in Pattern Recognition: A Review. International Journal of Advanced Research in Computer Science and Softwar Engineering, Volume 3, Issue 4, April 2013.

12. Vinita Dutt,Vikas Chaudhry and Imran Khan:Pattern Recognition: an Overview. American Journal of Intelligent Systems, 2012, 2(1): 23-27,DOI: 923/j.ajis.20120201.04.

13. Jie Liu, Jigui Sun and Shengsheng Wang:Pattern Recognition: An overview. IJCSNS International Journal of Computer Science and Network Security, vol. 6, no. 6, June 2006.

14. Anke Meyer -Base:Pattern Recognizing for Medical Imaging. Text book, ISBN: 0-12-4932908.

15. MIN720: Pattern Classification in Biomedical Applications.Course Lecture Notes, METU Informatics Institute, METU, 2010

16. Wikipedia Free Encyclopedia : www.wikipedia.com.

17. Neeraj Sharma and Lalit M. Aggarwall : Automated medical image segmentation techniques. J Med Phys. 2010 Jan-Mar; 35(1): 314.

18. Marko Wilke:Template-O-Matic: A toolbox for creating customized pediatric template. Volume 41, Issue 3, 1 July 2008, Pages 903-913. 